\title{
Qualite de vie des Patients ayant une Gonarthrose a Brazzavile, Congo
}

\author{
Lamini N'Soundhat N. E., \\ Malanda Nskiou Y. E., \\ Service de Rhumatologie,
}

Centre Hospitalier Universitaire de Brazzaville, République du Congo

Tiendrebeogo J.,

Ouedraogo D. D.,

Service de Rhumatologie,

Centre Hospitalier Universitaire de Bogodogo, Ouagadougou, Burkina Faso

\author{
Ntsiba H., \\ Bileckot R.,
}

Service de Rhumatologie,

Centre Hospitalier Universitaire de Brazzaville, République du Congo

Doi:10.19044/esj.2019.v15n24p90 ～URL:http://dx.doi.org/10.19044/esj.2019.v15n24p90

\section{Resume}

Objectif : Evaluer la qualité de vie des patients ayant une gonarthrose. Patients et Méthodes: Etude transversale, descriptive, menée dans le Service de Rhumatologie du CHU de Brazzaville, en deux étapes. La première a permis de colliger les dossiers des patients diagnostiqués selon les critères de l'ACR de 1986 et suivis pour une gonarthrose du $1^{\text {er }}$ janvier 2013 au 31 décembre 2017.La seconde consistait, après consentement éclairé, en la mesure de la qualité de vie à l'aide du questionnaire Arthrose des Membres Inférieurs et Qualité de vie (AMIQUAL) des patients identifiés. Elle s'est déroulée du 02 mai au 02 novembre 2018 (6 mois). Résultats : Nous avons inclus 103 femmes et 16 hommes (sexe ratio H/F de 1/6). L'âge moyen était de 56,1 +/- 9 ans (extrêmes 27 à 77 ans). La durée moyenne d'évolution était de 5,1 +/- 3,6 ans. La gonarthrose altérait de façon modérée la qualité de vie globale des patients. Les dimensions les plus touchées étaient les activités physiques et la douleur, avec respectivement $51,3 \%$ et $42,9 \%$ des patients compris entre 25 et 50 . Les scores moyens des items indépendants étaient respectivement de 35,3 +/- $24,4 \quad(\mathrm{n}=84)$ pour la dimension activité professionnelle, $36,2+/-20,8(\mathrm{n}=61)$ pour la relation de couple et $39,4+/-27,2$ $(\mathrm{n}=61)$ pour la sexualité. Conclusion : la gonarthrose affecte la qualité de vie globale des patients notamment dans les dimensions activités physiques et douleur. 
Mots clés : Arthrose, Genou, Gonarthrose, Qualité de vie, Brazzaville.

\title{
Quality of Life in Patients with Knee Osteoarthritis in Brazzaville, Congo
}

\author{
Lamini N'Soundhat N. E., \\ Malanda Nskiou Y. E., \\ Service de Rhumatologie,
}

Centre Hospitalier Universitaire de Brazzaville, République du Congo

Tiendrebeogo J., Ouedraogo D. D.,

Service de Rhumatologie,

Centre Hospitalier Universitaire de Bogodogo, Ouagadougou, Burkina Faso

Ntsiba $\mathrm{H}$.,

Bileckot R.,

Service de Rhumatologie,

Centre Hospitalier Universitaire de Brazzaville, République du Congo

\begin{abstract}
Objective: To evaluate the quality of life in patients with knee osteoarthritis. Patients and Methods: Cross-sectional, descriptive and analytical study, conducted in two steps in the Department of Rheumatology at the University Hospital of Brazzaville. The first step made it possible to collect data of patients diagnosed as having knee osteoarthritis according to the ACR criteria of 1986 and followed up from January 1st, 2013 to December 31st, 2017. After informed consent was obtained, the second step consisted in measuring the quality of life of patients using a specific quality-of-life questionnaire for knee and hip osteoarthritis OAKHQOL (OsteoArthritis of Knee Hip Quality Of Life). It took place from May 2nd to November 2nd, 2018 (6 months). Results: We included in this study 103 women and 16 men (sex ratio $\mathrm{F} / \mathrm{H}$ of 6/1). The average age was $56.1 \pm 9$ years (range 27 to 77 years). The mean evolution time was $5.1 \pm 3.6$ years. The knee osteoarthritis moderately impaired the overall quality of life of patients. The most affected dimensions were physical activity and pain, with respectively $51.3 \%$ and $42.9 \%$ of patients between 25 and 50. Mean scores for additional items were $35.3+/-24.4$, respectively, $(\mathrm{n}=84)$ for employment, $36.2+/-20.8(\mathrm{n}=61)$ for
\end{abstract}


relationship with the partner and $39.4+/-27.2(n=61)$ for sexuality. Conclusion: Knee osteoarthritis affects the overall quality of life of patients, especially in the physical activity and pain dimensions.

Keywords: Osteoarthritis, Knee, Quality of life, Brazzaville

\section{Introduction}

La gonarthrose est l'arthrose des membres la plus fréquente. Elle résulte de phénomènes mécaniques et biologiques qui déstabilisent l'équilibre entre la synthèse et la dégradation du cartilage et de l'os sous chondral (Sharma et al, 2006). Elle constitue, à l'heure actuelle, un véritable problème de santé publique, touchant environ 250 millions de personnes dans le monde (Breville et al, 2015). En Afrique Sub-saharienne, la gonarthrose représente entre 8 et $16 \%$ des consultations en Rhumatologie (Oniankitan et al, 2009 ; Doualla-Bija et al, 2014 ; Ouédraogo et al, 2014). Les principaux facteurs qui lui sont associés sont l'âge, le sexe féminin, l'obésité et les déformations axiales (Doualla-Bija et al, 2014; Mounach et al, 2008; Lamini N'Soundhat et al, 2017). Les patients sont souvent vus à un stade évolué de la maladie, rendant compte d'un retentissement fonctionnel important, en raison tant de la douleur que des fréquents troubles statiques (Ouédraogo et al, 2008 ; Oniankitan et al, 2014). L'impact de la gonarthrose sur la qualité de vie a été peu étudié en Afrique Sub-saharienne.

$\mathrm{Au}$ Congo, aucune donnée n'existe à ce sujet. En l'absence de possibilité de remplacement prothétique au cours des gonarthroses, la prise en compte de la qualité de vie s'impose pour mieux apprécier le vécu du patient, le retentissement de la maladie et adapter la prise en charge médicale (Kawano, 2015). Le but de notre travail était ainsi d'évaluer la qualité de vie des patients ayant une gonarthrose, suivis dans le service de Rhumatologie pour une gonarthrose.

\section{Patients et méthode :}

Il s'agissait d'une étude transversale, descriptive, menée dans le Service de Rhumatologie du Centre Hospitalier Universitaire de Brazzaville. Dans un premier temps, les dossiers des patients suivis pour une gonarthrose, entre le $1^{\text {er }}$ janvier 2013 et le 31 décembre 2017 (soit 4 ans), ont été colligés. Ceux dont le diagnostic de gonarthrose répondait aux critères de l'ACR de 1986 ont été retenus (Altman et al, 1986). A partir des données recueillies dans les dossiers, les patients ont été contactés individuellement par téléphone ou joints à leur domicile. Après consentement éclairé, les patients ont été vus en consultation dans le service de Rhumatologie du CHU, afin d'apprécier l'évolution clinique notamment le retentissement clinique selon le score algo-fonctionnel de Lequesne, para-clinique (stade radiologique selon la 
classification de Kellgren et Lawrence) (Kellgren et al, 1957) et de mesurer leur qualité de vie (QV). Les données sur la QV ont été recueillies au moyen du questionnaire «Arthrose des Membres Inférieurs et Qualité de vie » ou AMIQUAL (Rat et al, 2006; Rat et al, 2005). L'AMIQUAL, développé en France en 2005, est un instrument spécifique de mesure de la qualité de vie chez les patients atteints d'une gonarthrose ou d'une coxarthrose. Sa version 2.3 comporte 43 items, répartis en cinq dimensions, à savoir la dimension 'Activités physiques', la dimension 'Santé mentale', la dimension 'Douleur', la dimension 'Soutien social, la dimension 'Activités sociales' et 3 items indépendants, à savoir l'activité professionnelle, les relations de couple et la sexualité. L'AMIQUAL a été administré en français et en langues nationales (Linguala ou Kitouba) pour les participants non alphabétisés ou ne comprenant pas la langue française. La traduction du questionnaire en langue nationale a été effectuée par le Département de langues nationales de la Faculté des Lettres et Sciences Humaines de l'Université Marien NGOUABI. Pour chacune des dimensions, le score normalisé va de 0 à 100. Les scores normalisés sont définis tels que 0 correspond à la pire qualité de vie et 100 à la meilleure. Pour faciliter l'interprétation des résultats, nous avons réparti les scores de la manière suivante : score normalisé inférieur à 25 : altération très sévère de la qualité de vie ; score normalisé compris entre 25 et 50 : altération sévère de la qualité de vie ; score normalisé compris entre 50 et 75 : altération modérée de la qualité de vie, et score normalisé supérieur à 75 : altération légère de la qualité de vie. Pour les items indépendants, le score va de 0 à 100 , tels que 0 corresponds à une absence d'altération et 100 à une altération très sévère. La mesure de la QV s'est déroulée du 02 mai au 02 novembre 2018 (6 mois). Le logiciel SPSS 22 a permis l'analyse des données.

\section{Resultats:}

Sur 181 dossiers de gonarthrose colligés dans le service de Rhumatologie, 119 répondaient aux critères d'inclusion. Il s'agissait de 103 femmes et 16 hommes (sexe ratio $\mathrm{H} / \mathrm{F}$ de $1 / 6$ ). L'âge moyen des patients était de 56,1 +/- 9 ans (extrêmes 27 et 77 ans). La durée moyenne d'évolution de la gonarthrose était de 5,1 +/- 3,6 ans avec des extrêmes de 1 et 21 ans. Le principal symptôme était la douleur de genou, retrouvée chez 105 patients $(88,2 \%)$. Elle était bilatérale chez 52,4\% $(n=55)$ des patients douloureux. L'intensité moyenne de la gonalgie selon l'échelle visuelle analogique (EVA) était de 47,1 $\pm 12,7$ sur 100 avec des extrêmes de 20 et 70 sur 100 . Un vice architectural a été retrouvé chez 73 patients $(61,3 \%)$. Il s'agissait d'un genu valgum chez 37 patients $(50,7 \%)$ et d'un genu varum chez 26 patients (35,6\%). Il n'a pas été retrouvé de cas de genu recurvatum, ni de genu flessum. La gonarthrose était bicompartimentale chez 47 patients $(39,5 \%)$, 
tricompartimentale chez 42 patients $(35,3 \%)$ et unicompartimentale chez 30 patients $(25,2 \%)$. L'atteinte radiologique selon la classification de Kellgren et Lawrence était classée grade I dans 9,24 \%, grade II dans 36,97 \%, grade III $38,66 \%$ et grade IV dans $15,13 \%$ des cas. Le score moyen de l'indice algofonctionnel de Lequesne était de 9,8 $\pm 3,6$ avec des extrêmes de 2 et 16,5. Le retentissement fonctionnel était jugé très sévère chez 21 patients $(17,64 \%)$, sévère chez 34 patients $(28,57 \%)$, important chez 35 patients $(29,41 \%)$, moyen chez 19 patients $(15,96 \%)$ et minime chez 10 patients $(8,40 \%)$.

Le score normalisé moyen le plus élevé était celui de la dimension activités sociales 75,8 +/- 19 (extrêmes allant de 23,3 et 100). Tandis que le score normalisé le plus bas était celui de la dimension activités physiques soit 54,3 \pm 16 avec des extrêmes de 29,5 à 94,4 (Tableau I).

Tableau I : Scores normalisés des dimensions de la qualité de vie selon l'AMIQUAL

\begin{tabular}{ccccc}
\hline & Moyennes & Ecart-types & Minimum & Maximum \\
\hline Activités physiques & 54,3 & 16 & 29,5 & 94,4 \\
Santé mentale & 59,9 & 12,9 & 29,2 & 96,9 \\
Douleur & 57,6 & 16,8 & 30 & 92,5 \\
Soutien social & 72,9 & 13,9 & 40 & 97,5 \\
Activités sociales & 75,8 & 19 & 23,33 & 100 \\
\hline
\end{tabular}

Interprétation : de 0-25 (altération très sévère) ; de 25-50 (altération sévère); de 50-75 (altération modéré) au delà de 75 (altération minime)

Soixante-et-un patients avaient un score normalisé entre 25 et 50 pour les activités physiques, 51 patients avaient un score normalisé entre 25 et 50 pour la douleur,79 patients avaient un score entre 50 et 75 pour la santé mentale, 57 patients avaient un score normalisé entre 50 et 75 pour le soutien social et 64 avaient un score normalisé supérieur à 75 pour les activités sociales (Tableau II). 
Tableau II : Distribution des patients en fonction des scores normalisés des dimensions de la qualité de vie selon l'AMIQUAL

\begin{tabular}{|c|c|c|}
\hline & \multicolumn{2}{|l|}{ Total } \\
\hline & Effectifs (n) & Pourcentage $(\%)$ \\
\hline Activités physiques & 119 & 100 \\
\hline Inférieur à 25 (Très sévère) & 0 & 0 \\
\hline 25-50 (Sévère) & 61 & 51,3 \\
\hline 50-75 (Modérée) & 41 & 34,4 \\
\hline > 75 (Légère) & 17 & 14,3 \\
\hline Santé mentale & 119 & 100 \\
\hline Inférieur à 25 (Très sévère) & 0 & 0 \\
\hline 25-50 (Sévère) & 27 & 22,7 \\
\hline 50-75 (Modérée) & 79 & 66,4 \\
\hline > 75 (Légère) & 13 & 10,9 \\
\hline Douleur & 119 & 100 \\
\hline Inférieur à 25 (Très sévère) & 0 & 0 \\
\hline 25-50 (Sévère) & 51 & 42,9 \\
\hline 50-75 (Modérée) & 47 & 39,5 \\
\hline > 75 (Légère) & 21 & 17,6 \\
\hline Soutien social & 119 & 100 \\
\hline Inférieur à 25 (Très sévère) & 0 & 0 \\
\hline 25-50 (Sévère) & 10 & 8,4 \\
\hline 50-75 (Modérée) & 57 & 47,9 \\
\hline > 75 (Légère) & 52 & 43,7 \\
\hline Activités sociales & 119 & 100 \\
\hline < 25 (Très sévère) & 1 & 0,8 \\
\hline 25-50 (Sévère) & 15 & 12,6 \\
\hline 50-75 (Modérée) & 39 & 32,8 \\
\hline > 75 (Légère) & 64 & 53,8 \\
\hline
\end{tabular}

Les items Q12, Q22 et Q23 de l'AMIQUAL intéressent respectivement les activités professionnelles, les relations de couple et la sexualité. Le score moyen de l'item des activités professionnelles a été calculé 
pour les patients actifs. Le score moyen des items concernant les relations de couple et la sexualité n'a été calculé que pour les patients qui avaient un conjoint (mariés ou union libre). Les scores moyens des items indépendants sont présentés dans le tableau III.

Tableau III : Scores moyens des items indépendants de l'AMIQUAL

\begin{tabular}{cccccc}
\hline & Effectifs & Moyennes & Ecart-types & Minimum & Maximum \\
\hline Activités professionnelles & 84 & 35,3 & 24,4 & 0 & 80 \\
Relations couple & 61 & 36,2 & 20,8 & 0 & 80 \\
Sexualité & 61 & 39,4 & 27,2 & 0 & 90 \\
\hline
\end{tabular}

Interprétation : le score va de 0 à 100, tels que 0 corresponds à une absence d'altération et 100 à la pire altération

\section{Discussion}

La place de la gonarthrose en milieu rhumatologique tropical est bien connue de nos jours. Elle constitue un véritable problème de santé publique du fait de sa fréquence et de l'inaccessibilité du remplacement prothétique. Elle prédomine chez la femme d'âge mûre dans les séries africaines (Oniankitan et al, 2009 ; Doualla-Bija et al, 2014 ; Ouédraogo et al, 2014). La douleur est le principal symptôme d'appel. Les troubles de la statique sont fréquents, retrouvés dans 73,4 à 87,4 \% selon les séries (Lamini N'Soundhat et al, 2017; Ouédraogo et al, 2008; Oniankitan et al, 2014). En règle générale l'atteinte radiologique était sévère et le plus souvent bicompartimentale, témoignant du retard de prise en charge médical. Le retentissement fonctionnel est le plus souvent sévère dans les séries africaines en corrélation avec l'obésité (Lamini N'Soundhat et al, 2017) et/ou les déviations axiales (Ouédraogo et al, 2008).

L'appréciation de la qualité de vie dans notre série montre que la gonarthrose affecte celle-ci chez nos patients. L'altération de la QV est classique au cours de la gonarthrose et cela quelque soit l'outil psychométrique utilisé (Kawano et al, 2015; Nawito et al, 2018). En effet, Nawito et al en Egypte et Kawano et al au Brésil utilisant respectivement le questionnaire Nottingham Health Profile (NHP) et le questionnaire générique Short-Form Health Survey (SF-36) ont conclu à une altération de la qualité de vie des patients gonarthrosiques dans leur série respective. Cependant, elle semble varier selon les cultures et en fonction de l'adaptation de l'outil de mesure utilisé selon les régions. En effet, Serhier et al au Maroc (Serhier et al, 2012) rapportaient des résultats moins bons que ceux de notre étude, mais aussi moins bons que ceux obtenus au Burkina Faso et en Egypte (sauf pour les dimensions soutien social et activités sociales en Egypte) [Figure 1]. 

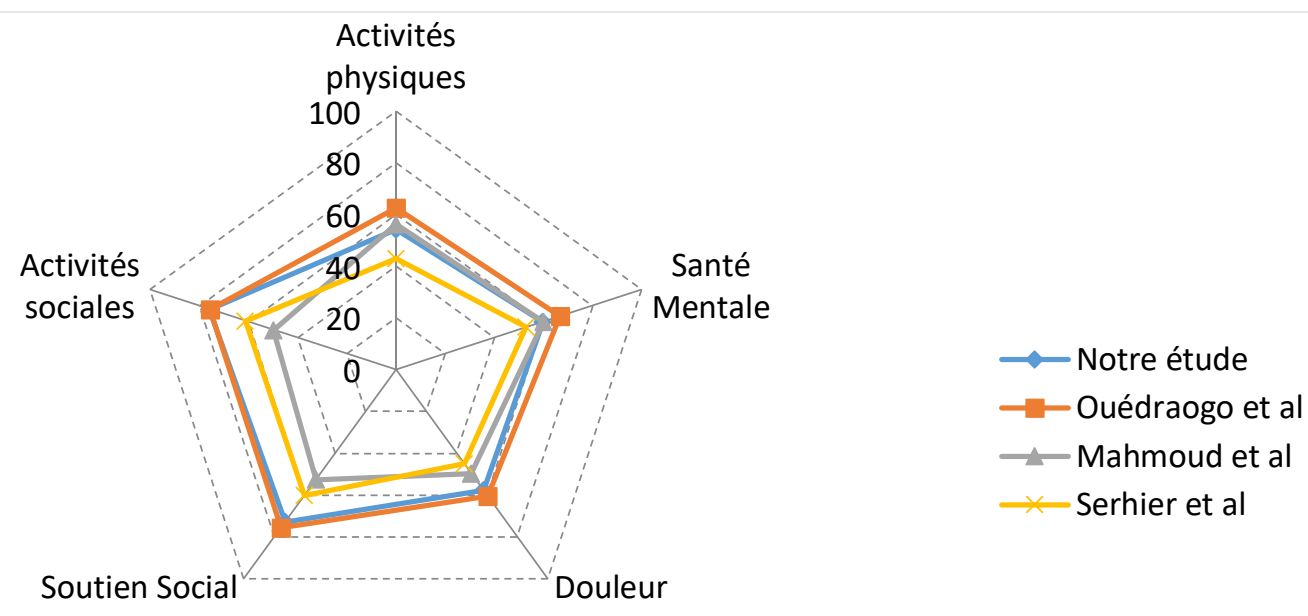

Figure 1: Résultats comparés de l'AMIQUAL par rapport aux séries africaines

Il en est de même, lorsqu'on compare nos résultats aux séries européennes et asiatiques [Figure 2], notamment la série de Rat et al, en France, de Gonzalez Saenz de Tejada et al, en Espagne, de Saffari et al, en Iran et de Kadir et al en Malaysie (Rat et al, 2006 ; Gonzalez Saenz de Tejada et al, 2011; Saffari et al, 2017; Kadir et al, 2018), exceptés pour la dimension 'soutien social'.

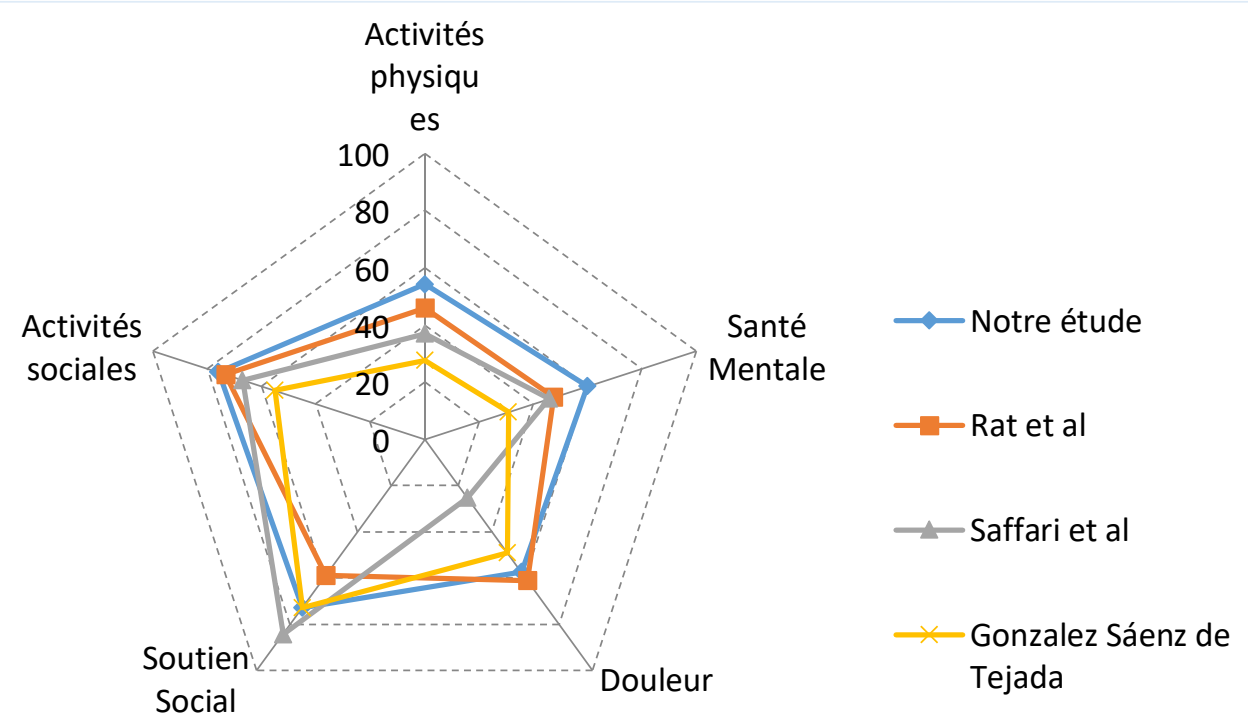

Figure 2 : Résultats comparés de l'AMIQUAL par rapport aux séries occidentales 
Il existe cependant un biais d'interprétation car L'AMIQUAL n'a pas été validé pour les populations d'Afrique sub-saharienne à la différence des populations européennes et asiatiques où les études de validation ont été menées (Rat et al, 2006; Kadir et al, 2018). La comparaison des résultats de cette étude avec ces séries est donc à relativiser et cela malgré la traduction $\mathrm{du}$ questionnaire dans nos langues nationales «linguala et kituba » pour faciliter sa compréhension par nos patients. Les scores normalisés moyens dans cette étude, montrent une qualité de vie altérée dans les dimensions 'activités physiques' et 'douleur'. Ces deux dimensions semblent être les dimensions les plus souvent affectées en Afrique. Il en est ainsi dans la série de Ouédraogo et al au Burkina Faso et Mahmoud et al en Egypte (Ouédraogo et al , 2014; Mahmoud et al, 2019). Dans les séries européennes et asiatiques, la dimension 'activité physique' est aussi la dimension la plus affectée quel que soit l'outil de mesure psychométrique (Rat et al, 2005; Gonzalez Saenz de Tejada et al, 2011; Saffari et al, 2017; Kadir et al, 2018). Cependant dans ces études, elle est la dimension la plus étudiée. En effet, le genou étant une articulation portante, il est évident que le retentissement sur la fonction reste l'élément d'appréciation le plus important à juger tant pour le retentissement fonctionnel, que pour la qualité de vie. En Afrique Sub-saharienne, le mode de vie favorise les contraintes aux genoux. Le mode de vie à hauteur de sol prédomine pour les activités de la vie domestique (l'hygiène corporelle, les activités ménagères notamment la cuisine, la prise de repas, les activités de manutention...). Ces habitudes de vie peuvent expliquer pourquoi la dimension 'activité physique' est la plus altérée. Ce qui renforce la place des facteurs socioculturels dans l'appréciation de la QV.

La sexualité, les relations de couple et les activités sociales semblaient être modestement affectées chez les patients de cette série. Cependant, il est difficile de confronter les scores des items indépendants aux résultats des autres études, dans la mesure où ils sont peu rapportés dans les séries (Serhier et al, 2012; Kadir et al, 2018; Ouédraogo et al, 2014; Mahmoud et al, 2019).

\section{Conclusion}

L'évaluation de la qualité de vie des patients gonarthrosiques à Brazzaville, au moyen du questionnaire Arthrose des Membres Inférieurs et qualité de vie (AMIQUAL) a permis de mettre en évidence une altération modérée de la qualité de vie globale des patients. Les scores les plus bas étaient retrouvés dans les dimensions 'activités physiques' et 'douleur'. En l'absence de possibilité de remplacement prothétique dans la gonarthrose au Congo, il est important qu'une place prépondérante soit accordée aux mesures physiques et réadaptatives mais aussi à l'éducation thérapeutique, dans la prise en charge médicale de cette affection. 
Conflit d'intérêts : aucun

\section{References:}

1. Sharma. L, Kapoor. D, Issa. S. (2006). Epidemiology of osteoarthritis: an update. Current Opinion in Rheumatology; 18(2): 147-56.

2. Breville. P, Le Quintrec. J L, Cadet. C, Verlhac. B, Vetel. J M, LevyRaynaud. O, Jeandel. C, Maheu. E. (2015). Le fardeau de l'arthrose. Les Cahiers de l'Année Gérontologique; 7(2) :45-51.

3. Oniankitan. O, Fianyo. E, Mijiyawa. M. (2009). Gonarthrose en consultation rhumatologique à Lomé (TOGO). Mali Médical; 25(2): 4-6.

4. Doualla-Bija. M, Luma. H N, Temfack. E, Gueleko. E T, Kempta. F, Ngandeu. M. (2014). Patterns of knee osteoarthritis in a hospital setting in sub-Saharan Africa. Clinical Rheumatology; 34(11): 194953.

5. Ouédraogo. DD, Ntsiba. H, Tiendréogo-Zabsonré. J, Tiéno. H, Bokossa. L I, Kaboré. F, Drabo. J. (2014). Clinical spectrum of rheumatologic diseases in a department of rheumatology in Ouagadougou (Burkina Faso). Clinical Rheumatology; 33(3): 385-9.

6. Mounach. A, Nouijai. A, Ghozlani. I, Ghazi. M, Achemlal. L, Bezza. B, Maghraoui. A E. (2008). Risk factors for knee osteoarthritis in Morocco. A case control study. Clinical Rheumatology; 27(3): 323-6.

7. Lamini N'Soundhat. N E, Mouelé Mboussi. P C, Nkouala-Kidédé. C D, Akoli Ekoya. O, Ntsiba. H. (2017). Knee osteoarthritis and risk factors associated. Open Journal of Rheumatology and Autoimmune Diseases; 7(3): 147-52.

8. Ouédraogo. DD, Séogo. H, Cissé. R, Tiéno. H, Ouédraogo. T, Nacoulma. I S, Drabo. Y J. (2008). Facteurs de risque associés à la gonarthrose en consultation de rhumatologie à Ouagadougou (Burkina Faso). Médecine Tropicale; 68(6): 597-9.

9. Oniankitan. O, Fianyo. E, Lama. K A K, Koffi-Tessio. V E S, Tagbor. K C, Houzou. P, Kakpovi. k, Mijiyawa. M. (2014). Facteurs de risque de la gonarthrose en consultation rhumatologique à Lomé (Togo). Revue Marocaine de Rhumatologie; 29: 28-31.

10. Kawano. M M, Araujo. I L A, Castro. M C, Matos. M A. (2015). Assessment of quality of life in patients with knee osteoarthritis. Acta Ortopédica Brasileira; 23(6):307-10.

11. Altman. R, Asch. E, Bloch. D, Bole. G, Borenstein. D, Brandt. K, Christy. W, Cooke. T. D, Greenwald. R, Hochberg. M, Howel. D, Kaplan. D, Koopman. W, Longley. S, Mankin. H, McShane. D J, Medsger. T, Meenan. R, Mikkelsen. W, Moskowitz. R, Murphy. W, Rothschild. B, Segal. M, Sokoloff. L, Wolfe. F. (1986). Development 
of criteria for the classification and reporting of osteoarthritis. Classification of osteoarthritis of the knee. Diagnostic and therapeutic criteria committee of the American rheumatism association. Arthritis and Rheumatism; 29(8): 1039-49.

12. Kellgren. J H, Lawrence. J S. (1957). Radiological assessment of osteo-arthrosis. Annals of the Rheumatic Diseases; 16(4):494-502.

13. Rat. A C, Pouchot. J, Coste. J, Baumann. C, Spitz. E, Retel-Rude. N, Baumann. M, Le Quintrec. J S, Dumont-Fischer. D, Guillemin. F; Groupe Qualité de Vie en Rhumatologie. (2006). Development and testing of a specific quality-of-life questionnaire for knee and hip osteoarthritis: OAKHQOL (OsteoArthritis of Knee Hip Quality Of Life). Joint Bone Spine; 73: 697-704.

14. Rat. A C, Coste. J, Pouchot. J, Baumann. M, Spitz. E, Retel-Rude. N, Le Quintrec. J S, Dumont-Fischer. D, Guillemin. F. (2005). OAKHQOL: a new instrument to measure quality of life in knee and hip osteoarthritis. Journal of Clinical Epidemiology; 58(1): 47-55.

15. Nawito. Z. O, El-Azkalany. G. S, El-Sayad. M. (2018). Nottingham health profile assessment of health-related quality of life in primary knee osteoarthritis patients: relation to clinical features and radiologic score. The Egyptian Rheumatologist; 40(4): 265-8.

16. Serhier. Z, Harzy. T, ELfakir. T, Diouny. S, El Rhazi. K, Bennani Othmani. M, Salmi. L R, Nejjari. C. (2012). Cross-cultural adaptation and validation of the knee and hip health-related quality of life (OAKHQOL) in a Moroccan Arabic-speaking population. Rheumatology International; 32(4): 1015-23.

17. Gonzalez Saenz de Tejada. M, Escobar. A, Herdman. M, Herrera. C, Garcia. L, Sarasqueta. C. (2011). Adaptation and validation of the Osteoarthritis Knee and Hip Quality of Life (OAKHQOL) questionnaire for use in patients with osteoarthritis in Spain. Clinical Rheumatology; 30(12): 1563-75.

18. Saffari. M, Emamai Meybodi. M K, Koenig. HG, Pakpour. A H, Rshidi Jahan. H. (2017). Psychometric examination of Paersian version of osteoarthritis knee and hip quality of life questionnaire. International Journal of Rheumatic Diseases; 20(11):1648-57.

19. Kadir. A A, Mohd Arif. M F, Ishak. A, Hassan. I I, Noor. N M. (2018). Adaptation and validation of the Malay version of the Osteoarthritis Knee and Hip Quality of Life Questionnaire among knee osteoarthritis patients. BioMed Research International; Article ID 4329751: 15 pages. https://doi.org/10.1155/2018/4329751.

20. Ouédraogo. DD, Tiendrébéogo/Zabsonré. J, Kenagnon. A D S, Kaboré. F, Compaoré. C, Drabo. Y J, Chevalier. X. (2014). Quality of life of patients with knee osteoarthritis with questionnaire 
OAKHQOL (OsteoArthritis of Knee Hip Quality of Life) in rheumatology consultation in Burkina Faso (West Africa). Open Journal of Rheumatology and Autoimmune Diseases; 4(4): 219-25.

21. Mahmoud. G A, Moghazy. A, Fathy. S, Niazy. M H. (2019). Osteoarthritis knee hip quality of life questionnaire assessment in Egyptian primary knee osteoarthritis patients: Relation to clinical and radiographic parameters. The Egyptian Rheumatologist; 41(1): 65-9. 